\title{
DOPPLER IMAGING RESULTS FOR AP STARS
}

\author{
ARTIE P. HATZES \\ McDonald Observatory, University of Texas at Austin \\ Austin, TX, 78712, USA
}

\begin{abstract}
Doppler imaging results for the surface abundance distribution on Ap stars are reviewed. The silicon distribution on these stars is often characterized by depleted spots near the magnetic poles and enhancements in ring-like structures at intermediate magnetic latitudes. These results are consistent with the predictions of diffusion theory. On two stars, $\gamma$ Ari and $\mathrm{CU}$ Vir, the silicon enhancements appear very close to a magnetic pole. This can be explained if silicon is enhanced where the magnetic field has its maximum horizontal field strength and these stars possess a decentered dipole field. Doppler images of abundance distributions on Ap stars may be used to accurately measure the obliquity angle and to estimate the decentering parameter for a dipole field. The chromium distribution on at least four Ap stars show a depleted band coincident with the magnetic equator and depleted spots at the magnetic poles. Such a distribution can only arise if the star has a predominantly quadrupole field, or if horizontal diffusion is playing a role in the distribution of elements.
\end{abstract}

\section{Introduction}

Magnetic Ap stars show anomalous abundances of the elements $\mathrm{Si}, \mathrm{Cr}, \mathrm{Fe}$, and rare earths that are distributed unevenly across the stellar surface. This inhomogeneous distribution also produces photometric and spectral variations. The magnetic nature of these stars was first discovered by Babcock (1947) who found that most most sharp-lined Ap stars have a longitudinal component to the magnetic field with a strength of a few kilogauss that varied with the rotation period of the star. This magnetic variability also extends to the more rapidly rotating Ap stars (Borra \& Landstreet 1980, hereafter $\mathrm{BL}$ ). These magnetic variations have been largely explained by the oblique rotator model - dipole magnetic field whose axis was inclined with respect to the rotation axis of the star (Stibbs 1950). 
Michaud (1970) first proposed what has become a widely accepted theory of the detailed mechanism of how the magnetic field on Ap stars can alter the local surface distribution of elements. These stars have a high effective temperatures and a stable outer atmosphere due to a weak outer convection zone; thus radiation and gravitational forces can influence the motion of elements. If the radiation force on an element exceeds gravity it rises in the atmosphere. Conversely, if gravity dominates it sinks. The magnetic field modifies this distribution by suppressing the motion of ions across magnetic field lines. The elemental distribution should thus reflect the surface field geometry. For example, if an element accumulates where the field lines are horizontal to the surface and is depleted where the field lines are vertical, then the surface distribution of this element in a dipole field should consist of an enhanced band at the magnetic equator and two depleted spots at the magnetic poles.

In spite of the decades of extensive observations and considerable theoretical progress in understanding the spectral and magnetic variations of Ap stars, many mysteries concerning these stars remain to be solved. What is the origin of the magnetic fields? Is it primordial or is it generated by a dynamo? Why are only $15 \%$ of B0 - F5 stars magnetic stars? Why are not all stars in this interval Ap stars? Is an Ap star part of the evolutionary process for a normal A star, and if not, what are the physical conditions that create a magnetic Ap star?

Doppler images of the surface abundance distribution of Ap stars can probe the exact relationship between magnetic field geometry and elemental distribution and thus provide a direct means of confirming the diffusion theory. This can also lead to a better understanding of how strong magnetic fields influence stellar atmospheres. Doppler images of a wide range of spectral types among the Ap class of stars and in different evolutionary states may ultimately resolve many of the mysteries of Ap stars.

\section{Doppler Images of Ap Stars}

The development of techniques for mapping the surface distribution of elements on a star has a long history dating to the first attempts by Deutsch (1958) who represented the distribution of local equivalent width on the star as an expansion of spherical harmonics. A system of equations relating such integrated quantities as radial velocity, equivalent width, and magnetic field variations were then solved for the expansion coefficients. This early Doppler imaging method had two drawbacks in that it failed to use the information inherent in the line shapes and the expansion series had to be truncated to only a few terms to make the problem tractable. These severely restricted the types of features that could be imaged. Falk 
\& Wehlau (1974) suggested that Deutsch technique could be improved by using the shapes of the spectral line profiles.

Pioneering work on a bona fide Doppler imaging technique, one that utilizes the actual line shapes of a rotationally broadened spectral line profile was made by Goncharsky et al. (1982). The inverse problem - the mathematical equation relating the surface distribution of elemental abundance to a time sequence of observed spectral line profiles - is ill posed, i.e. it is one that can have many solutions. Goncharsky et al. solved this equation using Tikhonov regularization. The misfit, $\chi^{2}$, between the observed and computed spectral lines was minimized subject to a constraint imposed by a smoothing function. In a slightly different approach to Doppler imaging, Hatzes et al. (1989) used maximum entropy principles to solve the inverse problem. In this instance the smoothness of an image was maximized subject to the constraint that the model spectral line profiles fit the observed profiles to within the level of the noise (i.e. constraint imposed by $\chi^{2}$ ).

Doppler images of the surface distribution of elements has been produced for a number of stars using both Tikhonov regularization and the maximum entropy method. In this short review it is impossible to discuss all these images. Rather, a few representative images will be shown and these will be discussed in the context of the oblique rotator model and diffusion theory. The Doppler images shown here were all derived using the maximum entropy method (MEM). This should not be considered an endorsement of this technique over Tikhonov regularization or other Doppler imaging techniques - they all should produce similar images. Rather MEM images were shown because they were readily available to the author.

\subsection{CU VIR}

Goncharsky et al. (1983) derived a silicon distribution using the Si II 3862 $\AA$ line and Tikhonov regularization. They found that silicon was enhanced in two spots separated by about $100^{\circ}$ in longitude centered on latitude $50^{\circ}$, near the location of the negative magnetic pole as determined by BL. Silicon was depleted in a region near the stellar equator and $180^{\circ}$ in longitude from the enhanced regions, i.e. near the location of the positive magnetic pole.

Figure 1 shows the silicon distribution for CU Vir derived using MEM and 13 observations of the Si II $6347 \AA$. The spectral observations were phased to the ephemeris $2,441,455.696+0.520675 E$. The $v \sin i$ was measured as $147 \mathrm{~km} \mathrm{~s}^{-1}$ and an inclination of $60^{\circ}$ was used in the modeling. In this and in all subsequent Doppler images the star is shown at eight equi-spaced phases and the skeleton star in the lower right corner shows the space orientation of the star. All regions with an enhanced abundance are shown as white, whereas depleted regions are shown as black. The terms 

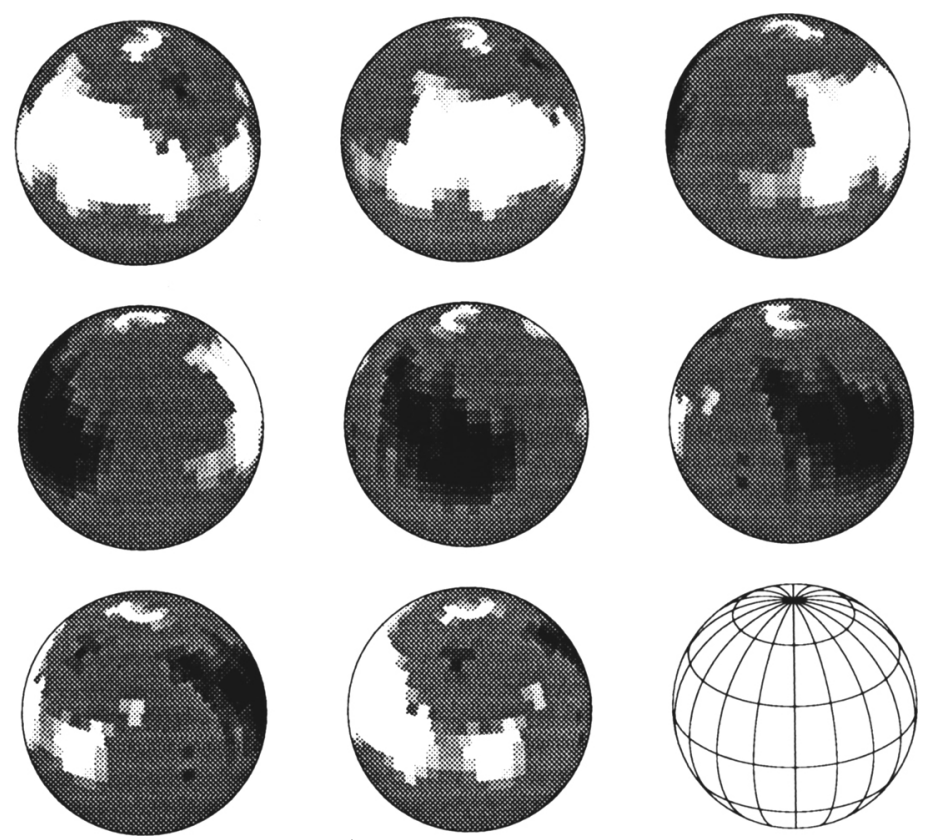

Figure 1. Doppler image of the silicon distribution on CU Vir derived using the Si II $6347 \AA$ line. White shows regions of enhanced silicon (maximum equivalent width, $W_{\lambda}$ $=600 \AA$ ) and black regions of relative depletions of silicon (minimum $W_{\lambda}=180 \mathrm{~m} \AA$ ). The mean equivalent width across the star is $330 \mathrm{~m} \AA$.

enhanced and depleted are with respect to the mean value across the star and are not to be interpreted as with respect to the solar value.

The Doppler image for CU Vir confirms the essential features of the Goncharsky et al. image. Silicon is depleted (local equivalent width, $W_{\lambda}$ $=180-200 \mathrm{~m} \AA$ ) in a spot located at phase 0.52 and latitude $+20^{\circ}$, near the position of the positive magnetic pole. By comparison the mean local equivalent width across the surface of the star is about $330 \AA$. There is a large elongated enhanced spot covering a region $100^{\circ} \times 40^{\circ}$ centered at phase 0.1 and latitude $25^{\circ}$. This feature corresponds to the two spots of enhanced abundance found by Goncharsky et al.

Hiesberger et al. (1995) derived a Doppler image of the He I distribution on CU Vir using the He I $4026 \AA$ line and Tikhonov regularization. They found that the silicon abundance varied by more than an order of magnitude across the stellar surface with the largest concentration nearly coincident with the depleted silicon region in Figure 1, and a less pronounced spot at phase $=0.80$. It appears that helium is underabundant where silicon is overabundant, a trend that was also seen in ET And (Piskunov et al. 1994). 


\section{2. $\gamma^{2}$ ARI}

$\gamma^{2}$ Ari is a $\mathrm{B} 9 \mathrm{pSiCr}$ star showing spectral variations in both silicon and chromium. A Doppler image of the silicon distribution was derived by Hatzes et al. (1989), again using the Si II $6347 \AA$ line $\left(v \sin i=64 \mathrm{~km} \mathrm{~s}^{-1}\right.$, $i=55^{\circ}$ ). This image is reproduced in Figure 2 (ephemeris $=2,446.279 .972$

$+1.60920 E)$. There is a prominent underabundant spot $\left(W_{\lambda}=30 \mathrm{~m} \AA\right)$ situated at phase 0.67 and latitude $35^{\circ}$. This spot is coincident in longitude with the negative magnetic pole as measured by BL. Surrounding this spot is an incomplete ring of enhanced silicon. This ring has a mean equivalent width of $300 \mathrm{~m} \AA$ and maximum value of $550 \mathrm{~m} \AA$ just below the underabundant spot. There is also another less pronounced underabundant spot near the expected longitudinal location of the positive magnetic pole.

The chromium distribution for this star is shown in Figure 3. This Doppler image was derived using 16 well-spaced observations of the $\mathrm{Cr}$ II $4824 \AA$ line. The distribution shows an elongated depleted region at phase 0.65 whose center is coincident with the depleted silicon spot. In spite of the similar location for these two features, the silicon and chromium appear to be spatially anti-correlated. The region with the least abundance of chromium coincides with those having the most abundant silicon (the low-latitude regions at phase 0.65 ). Furthermore, the center of the depleted silicon spot at this phase is coincident with the gap (i.e. a chromium abundance near the stellar average) in the depleted chromium spot. The anticorrelation can also be seen at phase 0.15 (the location of the positive magnetic pole). The chromium image shows no low-latitude depleted spot, but rather three spots of enhanced chromium surrounding the location of the depleted spot near the positive magnetic pole in the silicon image.

\section{3. $\theta$ AUR}

Silicon and chromium distributions were derived for this star using MEM (Hatzes 1991) and Tikhonov regularization (Rice \& Wehlau 1990). Figure 4 shows the silicon distribution derived using MEM and 14 observations of the Si II $6347 \AA$ line. Phases were calculated using the ephemeris JD = $2,446,337.972+3.6187 \mathrm{E}$. This distribution shows a small spot of silicon depletion $\left(W_{\lambda}=110 \mathrm{~m} \AA\right)$ centered at phase 0.55 and latitude $50^{\circ}$. BL determined a location of $\left(0.12,+39^{\circ}\right)$ and $\left(0.62,-39^{\circ}\right)$ for the location of the positive and negative poles respectively. Thus the depleted silicon spot is near the location of the negative magnetic pole, although at slightly higher latitude than the inferred magnetic pole. There also appears to be an enhanced ring of silicon with a radius of $50^{\circ}-90^{\circ}$ roughly centered on the depleted region. The average equivalent width in this ring is $350 \mathrm{~m} \AA$ and the maximum value of $400 \mathrm{~m} \AA$ occurs just below the depleted spot. 

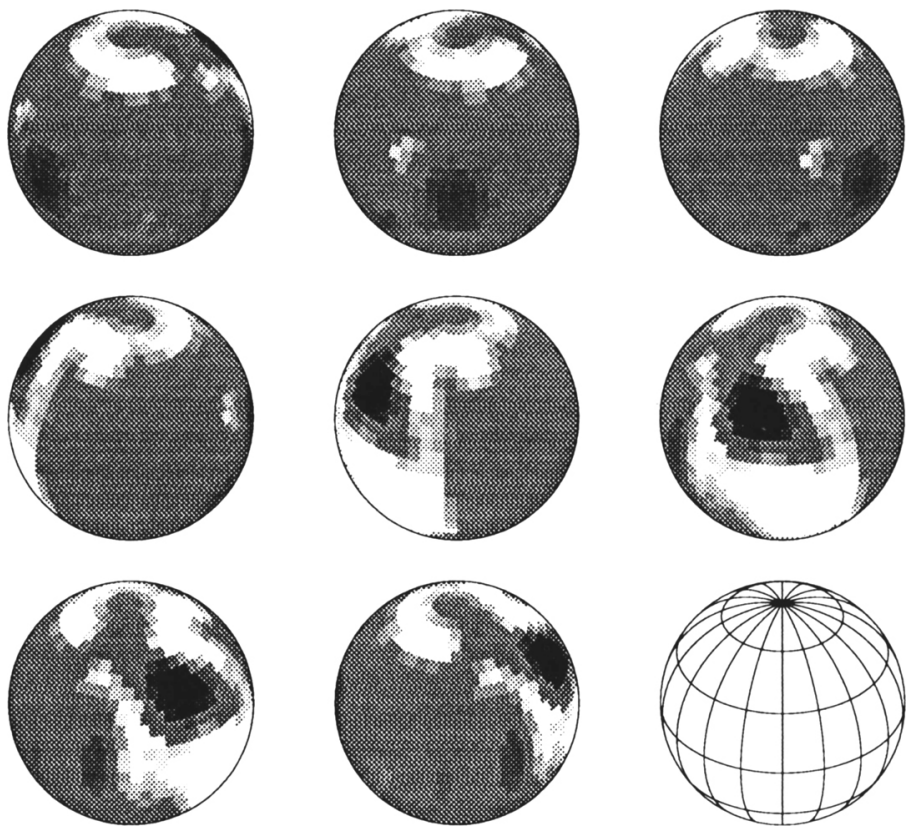

Figure 2. The silicon distribution on $\gamma^{2}$ Ari. Maximum $W_{\lambda}=550 \mathrm{~m} \AA$ (white), minimum $W_{\lambda}=5 \mathrm{~m} \AA$. Average $W_{\lambda}=170 \mathrm{~m} \AA$.
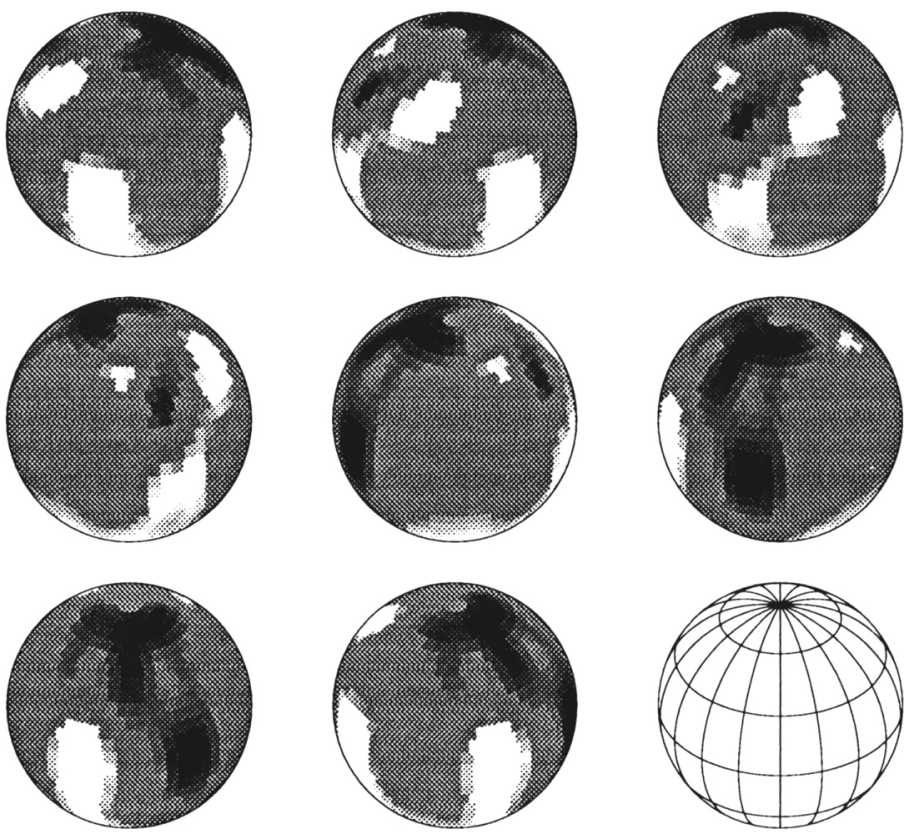

Figure 3. Doppler image of the chromium distribution on $\gamma^{2}$ Ari derived using the $\mathrm{Cr}$ II $4824 \AA$ line. Maximum $W_{\lambda}=200 \mathrm{~m} \AA$ (white), minimum $W_{\lambda}=140 \mathrm{~m} \AA$ (black), and mean $W_{\lambda}=280 \mathrm{~m} \AA$. 

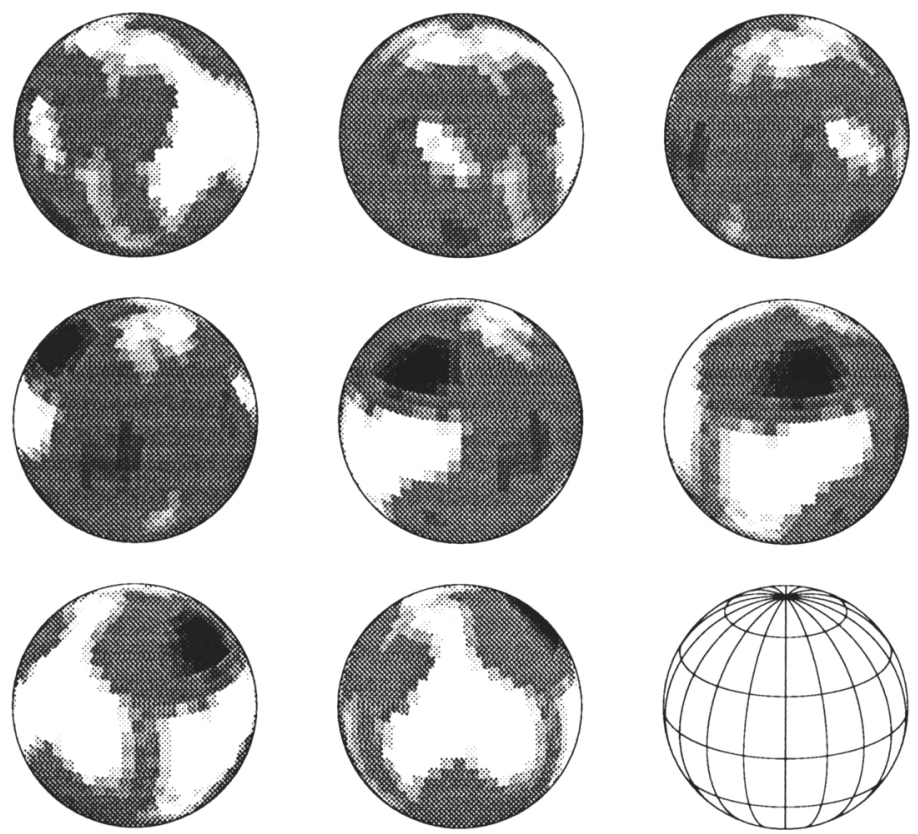

Figure 4. The silicon distribution $\theta$ Aur. maximum $W_{\lambda}=400 \mathrm{~m} \AA$ (white), minimum $W_{\lambda}=110 \mathrm{~m} \AA$ (black), and mean $W_{\lambda}=270 \mathrm{~m} \AA$.

This distribution is similar to the one derived by Rice \& Wehlau (1990) although they offered two interpretations for the silicon distribution. The first was that it consisted of a ring centered on $\left(0.65,+30^{\circ}\right)$ consistent with the interpretation of the MEM image. The second was that the enhancements formed two partial rings centered on $\left(0.12,+30^{\circ}\right)$ and $\left(0.62,-30^{\circ}\right)$.

Figure 5 shows the MEM Doppler image for the chromium distribution for this star derived using twenty observations of the $\mathrm{Cr}$ II $4824 \AA$. It consists of a large depleted spot $\left(W_{\lambda}=20 \mathrm{~m} \AA\right)$ spatially coincident with the depleted silicon spot. There are several small spots of enhanced abundance surrounding this depleted spot, but the largest concentration of silicon appears centered at the equator and phase 0.88 , very near the expected location of the positive magnetic pole. Another prominent feature of this image is the band of depleted chromium which seems to be coincident with the magnetic equator. Depleted bands have also been seen in the chromium images for 3 other stars: $\epsilon$ UMa, 45 Her, and $\omega$ Her (Hatzes 1991). In the case of $\epsilon$ UMa magnetic field measurements (Bohlender et al. 1990; Donati et al. 1990) have determined that the depleted band coincides with the magnetic equator.

Chromium images for $\theta$ Aur derived using Tikhonov regularization and the Cr II lines at $4901 \AA$ and $5508 \AA$ have also established that chromium 

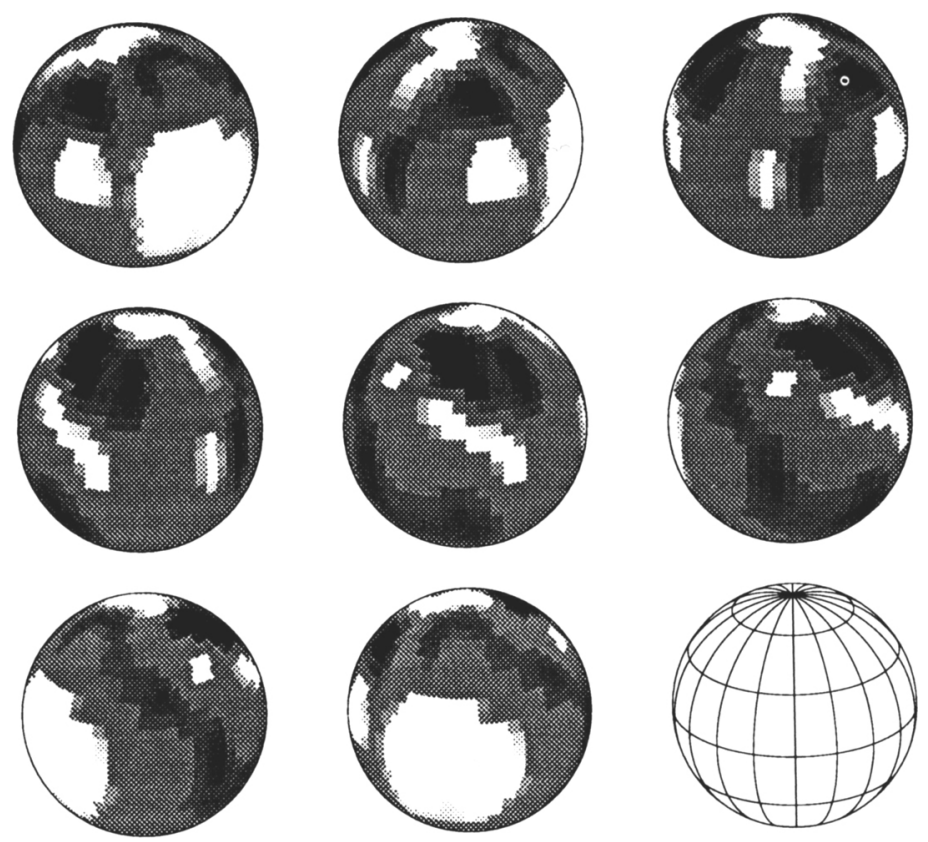

Figure 5. Doppler image of the chromium distribution $\theta$ Aur. Maximum $W_{\lambda}=250 \mathrm{~m} \AA$ (white), minimum $W_{\lambda}=20 \mathrm{~m} \AA$ (black), and mean $W_{\lambda}=100 \mathrm{~m} \AA$.

depletions on $\theta$ Aur occur at the magnetic equator while chromium enhancements occur at intermediate magnetic latitude (Rice \& Wehlau 1990). The strongest enhancements of chromium occurred at a location consistent with the low-latitude feature at phase 0.88 in Figure 5 . They also derived an iron image which showed an enhanced spot at this location.

Rice \& Wehlau (1991) determined the actual abundance values for silicon and chromium on $\theta$ Aur and found that they ranged from solar to 30 times the solar value. This means that the depleted regions shown in Figures 4 and 5 represent the solar value and are not true depletions.

\subsection{ORI}

Twenty-seven observations of the $\mathrm{Si}$ II $6347 \AA$ line were used to derive the MEM Doppler image shown in Figure 6. A $v \sin i$ of $50 \mathrm{~m} \mathrm{~s}^{-1}$ and an inclination of $70^{\circ}$ were used in deriving this image. Phases were calculated according to $\mathrm{JD}=2,444,280.9955+4.6398 E$.

The silicon distribution shows a large depleted spot in the shape of a vertical band centered on phase 0.63 . This band appears to have a gap (i.e. a region of "normal" equivalent width) at its center reminiscent of the chromium feature found on $\gamma^{2}$ Ari. If this feature is indeed part of a vertical band, then part of this band may extend over the rotation pole. However, 

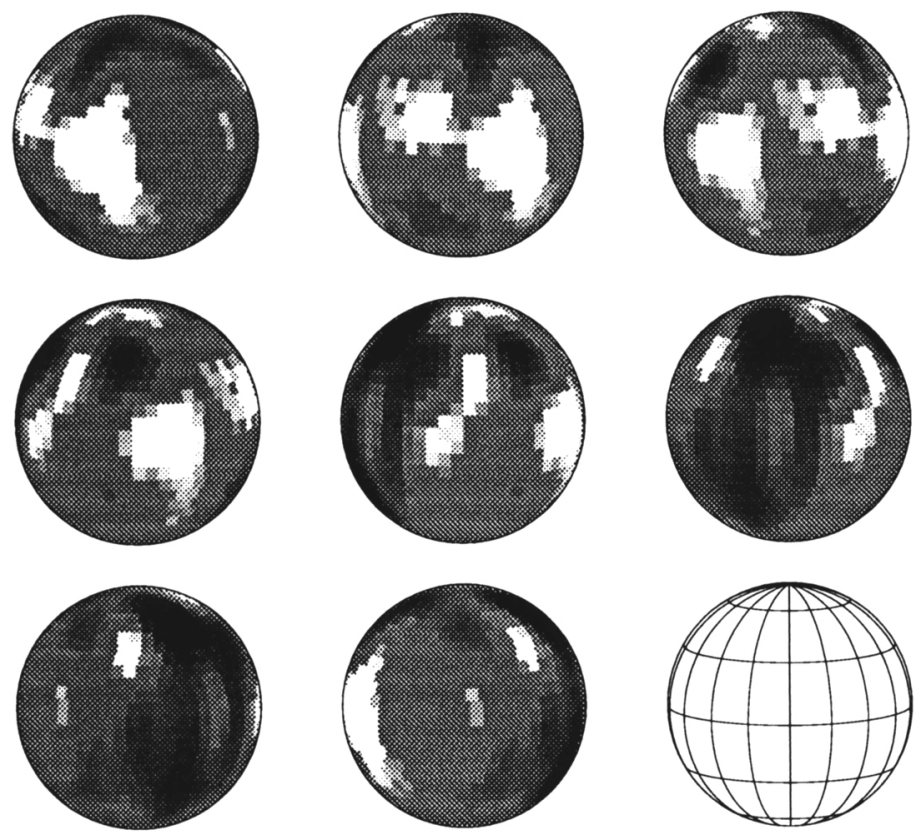

Figure 6. The silicon distribution 11 Ori. Maximum $W_{\lambda}=320 \mathrm{~m} \AA$ (white), minimum $W_{\lambda}=65 \mathrm{~m} \AA$ (black), and mean $W_{\lambda}=190 \mathrm{~m} \AA$.

mirroring due to the high inclination of the star may account for much of this band-like structure. There is also a depleted spot at latitude $40^{\circ}$ which passes the line-of-site 0.25 in phase prior to the passage of the large bandlike structure. Regions of enhanced silicon surround the depleted spot at phase 0.4 and a low-latitude depleted spot at phase 0.125 .

\section{Interpretation of the images}

If the magnetic field dictates the surface distribution of elements on Ap stars, then one should expect to see abundance features in the form of rings marking the magnetic equator or spots marking the magnetic poles. To a large extent this is what is seen in the Doppler images, although the rings are often incomplete and patchy. Depleted silicon spots occur at the magnetic poles of $\gamma^{2}$ Ari, CU Vir, and $\theta$ Aur. Enhanced silicon rings can be seen in the Doppler images of $\gamma^{2}$ Ari and $\theta$ Aur. Depleted bands of chromium seem to be coincident with the magnetic equator in $\theta$ Aur and $\epsilon \mathrm{UMa}$. Are these results consistent with diffusion theory, and if so, what can Doppler images tell us about the surface precise magnetic field geometry? 


\subsection{SILICON}

Theoretical work by Vauclair et al. (1979) and Alecian \& Vauclar (1981) on the diffusion of silicon suggests that this element in its ionized state should diffuse downwards in the atmosphere of Ap stars. One should thus see relative silicon depletions at the magnetic poles and the Doppler images confirm this. Their work also shows that since neutral silicon diffuses upwards, the overall silicon abundance should be enhanced in regions where the magnetic field is horizontal, i.e. the magnetic equator. Although silicon enhancements occur close to the magnetic equator of $\theta$ Aur, in other stars the region of silicon enhancements occur very near the location of a magnetic pole and far from the assumed location of the magnetic equator (for a centered dipole field).

Assuming that silicon is indeed enhanced where the field lines are horizontal, then two mechanisms can move the loci of horizontal field vectors closer to one magnetic pole: decentered dipoles or a combination of dipole plus quadrupole field. The addition of a quadruple component, however, can move the region of horizontal field lines no closer than $45^{\circ}$ from the magnetic poles, the loci of horizontal field angles for a pure quadrupole field. Decentered dipoles, on the other hand can have large horizontal field strengths quite close to a magnetic pole.

Figure 7 shows a "model" surface distribution for the magnetic field on CU Vir using a decentered dipole. White regions represent those places on the star having the maximum horizontal field strength. Dark regions represent regions of the star where the horizontal field strength is zero, i.e. where the field lines are vertical (to within a few degrees). An obliquity angle of $\beta=70^{\circ}$ for a dipole placed at $(x, y, z)=(-0.17,0.02,-0.06)$ was used, where $z$ is along the rotation axis and $x$ is in the direction of the observer at phase 0.0 . These values for the position of the dipole provide decentering primarily along the magnetic axis and in the direction of the positive pole at phase 0.5 . This field geometry qualitatively is able to reproduce the observed silicon distribution. The regions of enhanced silicon should appear as a band or elongated spot centered near phase 0.0 since low-latitude regions of the ring should not be well reconstructed. The area marking the vertical field lines (where silicon should be depleted) is large near the positive magnetic pole at phase 0.5 and small at the location of the negative magnetic pole. The small area of this latter spot coupled with the fact that it is at low-latitudes and in close proximity to the regions of enhanced silicon can easily explain why a depleted silicon spot is not seen at this location.

The line in Figure 8 is the predicted effective magnetic field measurements using the dipole configuration of Figure 7 and a dipole strength of 

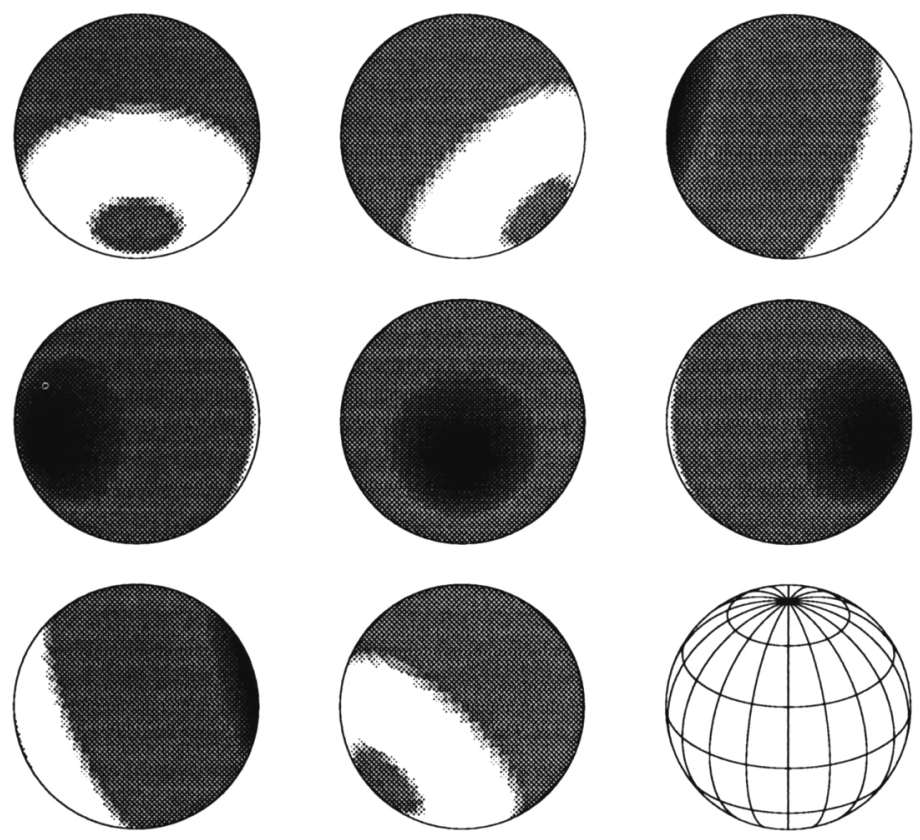

Figure 7. The surface magnetic field geometry on CU Vir using a decentered dipole. White regions mark where the field has a maximum horizontal field strength and black marks regions where the field lines are vertical with respect to the surface.

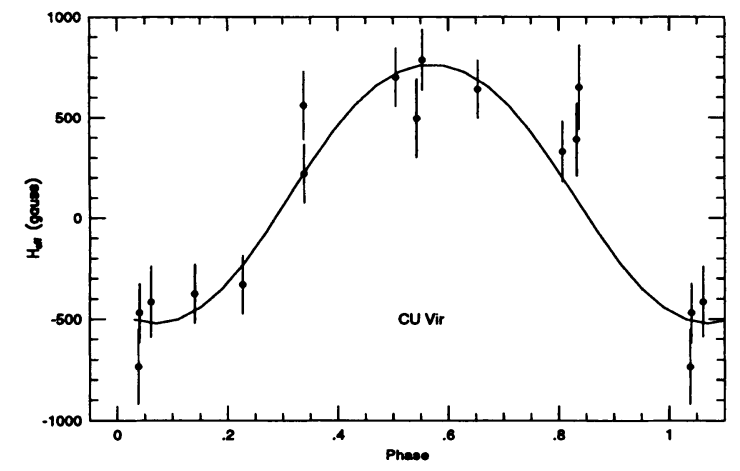

Figure 8. The predicted effective magnetic field strength (line) from the decentered dipole for CU Vir. The points are the measurements from BL.

3200 gauss. The points represent the magnetic field measurements of $\mathrm{BL}$ phased to the ephemeris of the Doppler image.

Figure 9 shows the magnetic field "model" for the silicon Doppler image for $\gamma$ Ari. Once again white regions represent the loci of maximum horizontal field strength while the black regions indicate the region where the field vectors are nearly vertical to the surface. The dipole parameters used to de- 

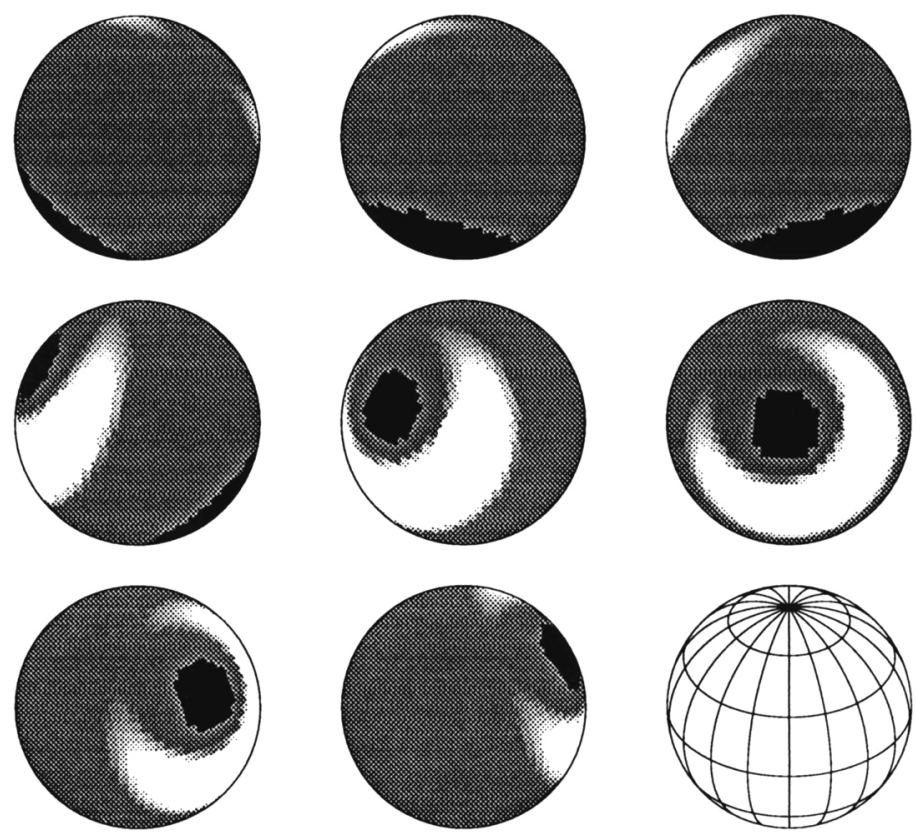

Figure 9. The distribution of surface magnetic field for $\gamma^{2}$ Ari. White marks the location of maximum horizontal field strength and black marks regions of vertical field lines.

rive this surface field distribution was $(\beta, x, y, x)=\left(40^{\circ}, 0.17,-0.06,0.08\right)$. Again, this surface magnetic field distribution qualitatively reproduces the major features of the silicon distribution (Figure 2). The ring of maximum horizontal field strength (i.e. silicon enhancements) lies close to the small spot marking the regions of vertical field lines (i.e. depleted silicon). Note that the strength of magnetic field along the ring is non-uniform, much like the silicon ring, and it has its maximum strength in a region just below the "depleted" spot, the same location as the maximum silicon abundance. This decentered dipole model can even explain the "gap" seen in the silicon ring. The opposite magnetic pole has a very large area, but occurs at a very low-latitude, regions that are not well reconstructed with the Doppler imaging technique. Also, Doppler imaging tends to reconstruct very low-latitude features at a higher latitude than they actually occur. Both of these may explain for the weakness of the second depleted spot at phase 0.13 and the fact that it appears at high latitude.

The expected magnetic field variations for this dipole configuration is shown as a line in Figure 10 along with the effective magnetic field variations of BL. A dipole strength of 4200 gauss was used in computing these variations.

It is encouraging that at least in the case of CU Vir and $\gamma^{2}$ Ari that decentered dipoles can explain the silicon distribution and the magnetic 


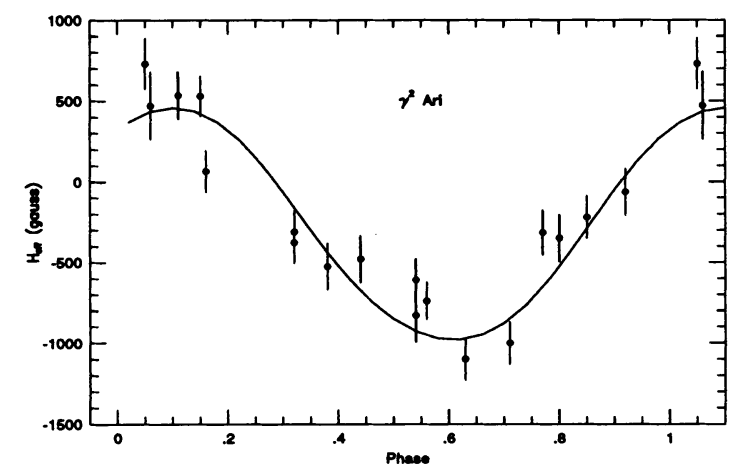

Figure 10. The predicted effective magnetic field strength (line) from the decentered dipole for $\gamma^{2}$ Ari. The points are the measurements from BL.

field measurements. Although there is hope that one day abundance maps on their own can be used to infer the magnetic field geometry on Ap stars, one should be cautious in doing so since effects other than magnetic field geometry may alter the abundance distribution. In particular, Megessier (1984) has proposed that horizontal diffusion may play an important role in determining the surface distribution of elements on evolved stars. She has argued that silicon may originally be enhanced at the star's magnetic equator, but as the star ages this ring of enhancement slowly diffuses horizontally leaving a depleted region at the equator and an enhanced ring that is closer to one magnetic pole. Perhaps this explains why the enhanced silicon regions on $\mathrm{CU}$ Vir and $\gamma^{2}$ Ari lie so close to one magnetic pole. If this is the case, then the decentered dipole model for these stars are just a convenient parameterization of the observed silicon distribution and have no relationship to the true magnetic field geometry.

\subsection{CHROMIUM}

The chromium images for $\theta$ Aur and $\epsilon$ UMa show that chromium is depleted at the magnetic equator. Depleted bands are also seen on $45 \mathrm{Her}$ and $\omega \mathrm{Her}$, but field measurements have yet to establish if these are indeed coincident with the magnetic equator. These chromium images also show isolated depleted spots that are separated by 0.25 in phase from the depleted band (coincident with a magnetic pole for $\epsilon \mathrm{UMa}$ and $\theta$ Aur) and that are often surrounded by enhanced chromium spots in a ring $\approx 45^{\circ}$ from the depleted spot. If chromium is depleted where the magnetic field lines are vertical and enhanced where the field lines are horizontal, then these distributions are precisely what is expected from an almost pure quadrupole field. However, magnetic field measurements for both $\theta$ Aur and $\epsilon$ UMa are adequately 
modeled with a pure dipole field. Furthermore, if $\theta$ Aur has a quadrupole field, why is this not reflected in the silicon distribution as well? Instead, the silicon distribution is consistent with a pure dipole field (i.e. no depleted bands). Although it cannot be completely refuted, the hypothesis that we are seeing quadrupoles on these stars seems unlikely.

Another possibility is that the depleted bands result from horizontal diffusion. Perhaps chromium was initially enhanced at the magnetic equator, but the stars showing depleted bands are old enough such that chromium has had time to drift toward a magnetic pole, thus leaving a depleted region at the equator. The differences seen between the silicon and chromium Doppler images may arise from the different timescales for the elements to diffuse horizontally.

In fact chromium on $\gamma^{2}$ Ari and silicon on 11 Ori may provide some evidence to support horizontal diffusion as a viable mechanism for altering the abundance distribution on Ap stars. If one interprets the large depleted silicon feature at phase 0.62 on 11 Ori as a vertical band, then silicon on this star mimics the distribution of chromium on $\theta$ Aur and other chromium stars. If so, then this phase should represent the magnetic equator. More likely, the silicon distribution is more similar to chromium on $\gamma^{2}$ Ari in that it has depleted spots straddling the location of the magnetic pole. If so, then phase 0.62 represents the magnetic pole of 11 Ori. Regardless of the interpretation, it seems that silicon on 11 Ori is distributed like chromium on other stars. Coincidentally, the radius of 11 Ori is anomalously large (> $4.6 R_{\odot}$ as inferred from the period and $v \sin i$ ) indicating that this star is possibly evolved. Chromium on $\gamma$ Ari also looks different from the chromium distribution found on other stars. Recent Doppler images of 17 Com show that chromium is enhanced in a ring surrounding a magnetic pole (Rice \& Wehlau 1994) which is also distinctly different than chromium images for $\theta$ Aur and $\epsilon$ UMa. Possibly these differences are due to a difference in evolutionary state of these stars.

\subsection{FUTURE DIRECTIONS FOR DOPPLER IMAGING OF AP STARS}

Although Doppler images of the distribution of elements on Ap stars are providing valuable clues as the magnetic field geometry on theses stars and tests for the diffusion mechanism, there are several key areas where progress can make Doppler images a more effective tool for studying Ap stars.

- More magnetic field measurements as well as the application of Zeeman Doppler imaging to Ap stars. These measurements are crucial to relating abundance features to the magnetic field geometry which is the key to interpreting the abundance images.

- Understand the effects of horizontal diffusion. 
It is not known what role, if any, horizontal diffusion plays in determining the observed abundance distribution on an Ap star. Doppler images of Ap stars with the same spectral type in clusters of known ages may help establish the effects of this process.

- Image a variety of spectral lines.

With the advent of echelle spectrographs which can provide large wavelength coverage at high resolution in a single exposure it is now possible to obtain simultaneously spectral data on a large number of spectral lines of different atomic species. The distribution of these various elements with respect to the magnetic field can then be compared to the predictions of diffusion theory.

- Diffusion calculations for other elements

Calculations of the diffusive behavior of other elements (chromium for instance) in the presence of a magnetic field and including the effects of horizontal diffusion are crucial for aiding in the interpretation and utilization of Doppler images.

Acknowledgements: This review is dedicated to the memory of Prof. William H. Wehlau who made significant contributions in surface imaging of Ap stars. The field has suffered a great loss in his passing. This work was supported by NSF grant AST-9315115.

\section{References}

Alecian, G. \& Vauclair, S. 1981, $A \& A, 101,16$.

Babcock, H.W. (1947), ApJ, 105, 105

Bohlender, D.A. \& Landstreet, J.D. (1990), ApJ Letters, 358, L25

Borra, E.F. \& Landstreet, J.D. (1980), ApJS, 42, 421 (BL)

Deutsch, A.J. (1958), Handbk. Phys., 51, 689

Donati, J.-F., Semel, M. \& del Toro Iniesta, J.C. 1990, $A \& A, 233,17$.

Falk, A.E. \& Wehlau, W.H. 1974, ApJ, 192, 409.

Goncharsky, A.V, Stepanov, V.V., \& Yagola, A.G. (1982), Astr. Zh., 26, 690

Goncharsky, A.V, Ryabchikova, T.A., Stepanov, V.V., Khokhlova, V.L., \& Yagola, A.G. (1983), Astr. Zh., 60, 83

Hatzes, A.P. (1990), MNRAS, 245, 56

Hatzes, A.P. (1991), MNRAS, 248, 487

Hatzes, A.P., Penrod, G.D., \& Vogt, S.S. (1989), ApJ, 341, 456

Hiesberger, F. et al., $A \& A, 296,473$

Michaud, G. (1970), $A p J, 160,640$

Mégessier, C. 1984, $A \mathscr{E} A, 138,267$.

Piskunov,N., Ryabchikova, T.A., Kusching, R., Weiss, W.W. (1995),

Rice, J.B. \& Wehlau, W. (1990), $A \& A, \mathbf{2 3 3}, 512$.

Rice, J.B. \& Wehlau, W. (1991), $A \& A, 246,195$.

Rice, J.B. \& Wehlau, W. (1994), $A \& A, 291,825$.

Stibbs, D.W.N. 1950, MNRAS, 110, 395.

Vauclair, S., Hardorp, J. \& Peterson, D. 1979, ApJ, 227, 526. 


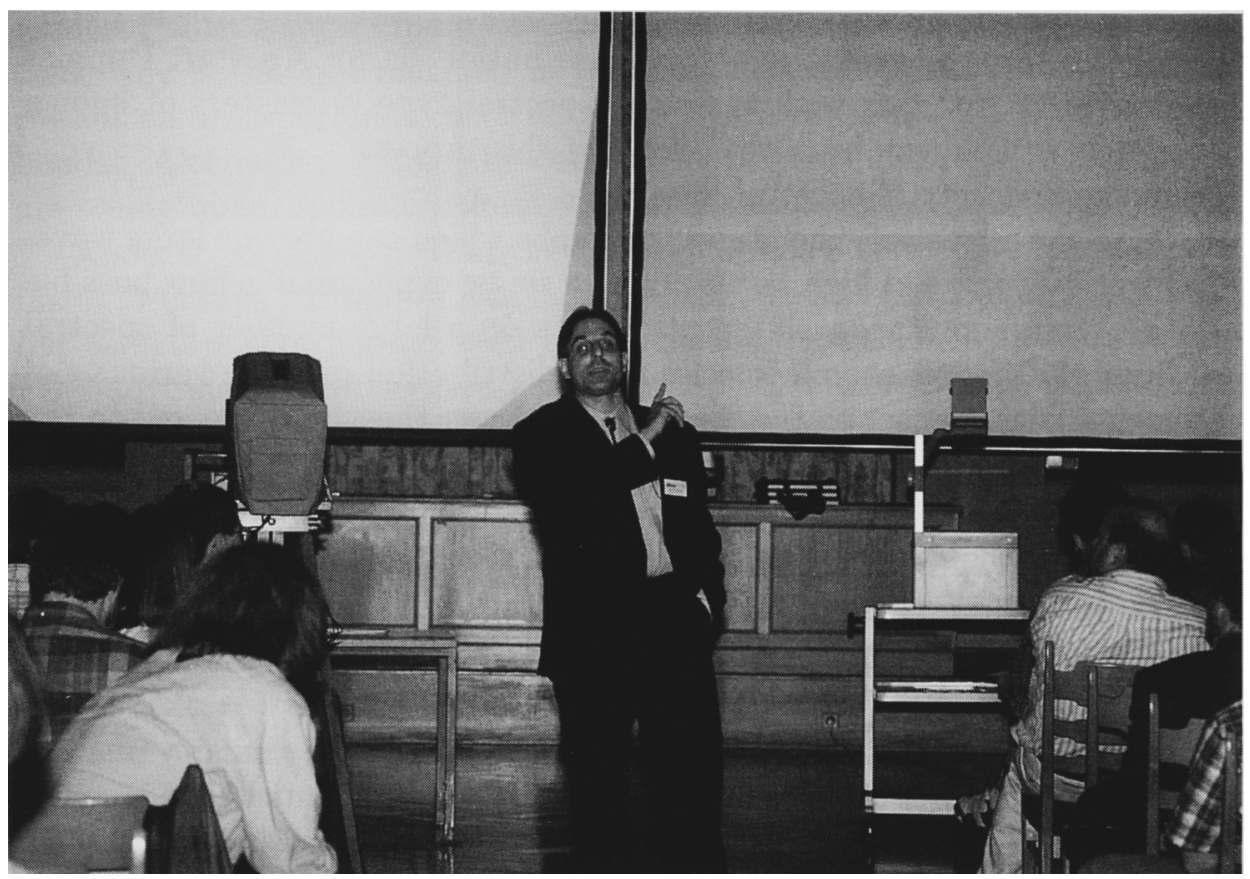

Artie Hatzes at work ...

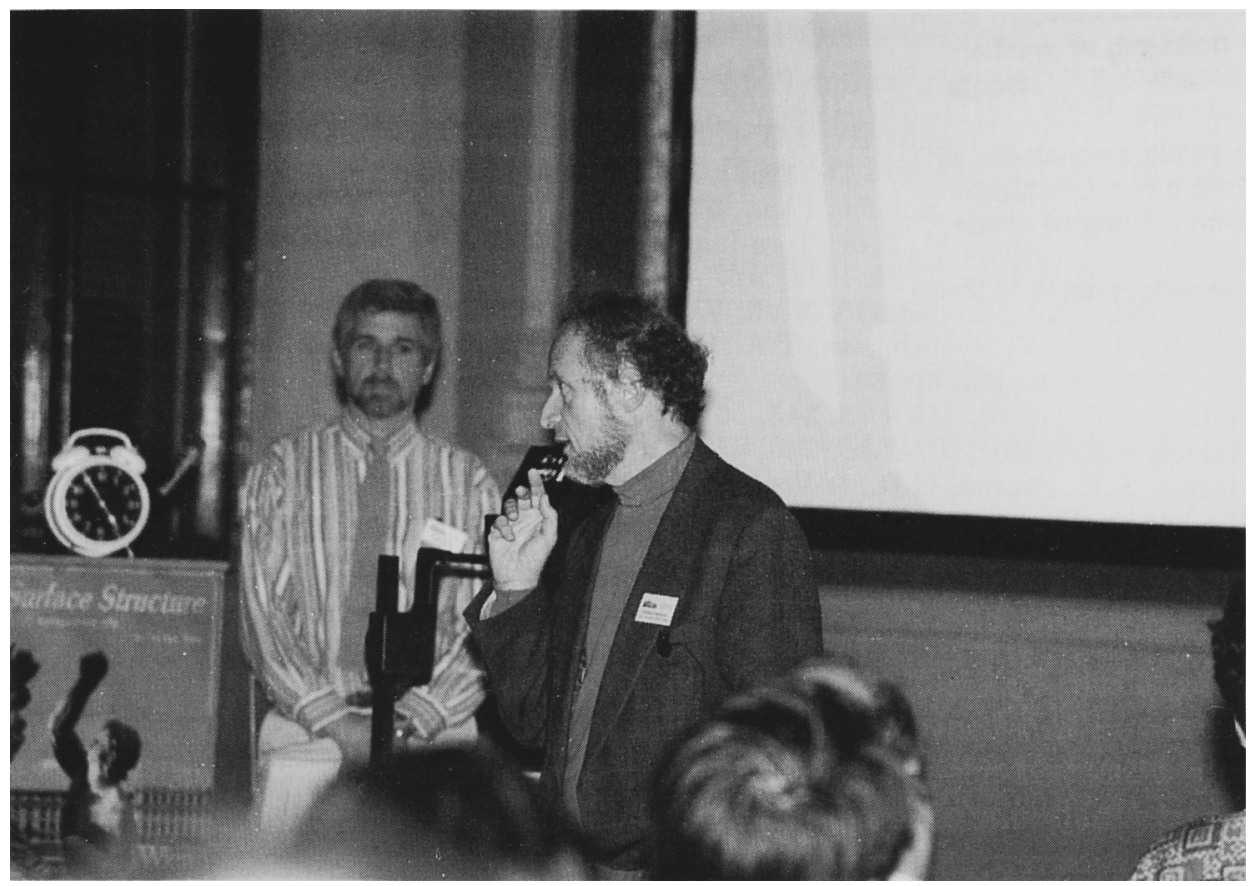

The session chairman (Steve Vogt, in the background - with tie!) is already counting George Michaud's remaining speaking time. 